\title{
Clinical Features, Echocardiographic Findings, Management and Outcome in a Young Male Patient with Atrial Myxoma: A Rare Primary Cardiac Tumor
}

Mohamed Farah Yusuf Mohamud ( $\nabla$ m.qadar59@gmail.com )

Mogadishu Somali Turkish Training and Research Hospital https://orcid.org/0000-0002-3719-3729

Said Abdirahman Ahmed

Mogadishu Somali Turkish Training adn research Hospital

Mohamed Abdullahi Mohamud

Mogadishu Somali Turkish Training and Research Hospital

\section{Case report}

Keywords: Atrial myxoma, Echocardiography, Electrocardiography, Surgery, Tumor,

Posted Date: September 29th, 2021

DOI: https://doi.org/10.21203/rs.3.rs-898467/v1

License: (c) (i) This work is licensed under a Creative Commons Attribution 4.0 International License.

Read Full License 


\section{Abstract}

Primary cardiac tumors in pediatric are rare and mostly benign. Atrial Myxoma is the most common primary tumor of the heart, which is usually involved in the left atrium. Surgical treatment is recommended when the patients develop symptoms or hemodynamically is impaired. Cardiac Myxoma can cause left atrial obstruction and systemic embolization. So early diagnosis with surgical intervention is the definitive management of the tumor and should not be delayed. Here we present a case of a 23year old male who admitted to the emergency department with syncope and dyspnea for 1 day ago. The tumor was removed surgically; pathologic examination confirmed the diagnosis of Myxoma.

\section{Introduction:}

Cardiac tumors are classified into primary cardiac tumors that can be benign or malignant neoplasm that may arise from any tissue of the heart with an autopsy incidence of $0.001-0.03 \%$, and Secondary or metastatic cardiac tumors that are 30 times more common than the primary neoplasm with an autopsy incidence of $1.7-14 \% .2[1,2]$. We report here a case of a 23-year old male with primary Atrial Myxoma that successfully removed in a surgical approach.

\section{Case Report:}

A 23 years old male was admitted to the emergency department with syncope and dyspnea on exertion for 1 day ago. He had no risk factors for atherosclerosis. The patient stated that he developed dyspnea while he is sweeping and lost his consciousness. The patient's vital signs were unremarkable except for mitral regurgitation on cardiac auscultation. Laboratory investigations were in a normal range. Electrocardiography revealed left atrial enlargement ( fig-1). A tremendous, nonhomogeneous mass at the level of the mitral valve was seen on the patient's Echocardiography which was respectively diagnosed as atrial Myxoma (fig-2a, 2b\& 2c). Fortunately, the left ventricular function and wall motion were normal. The patient was referred to another hospital that has a cardiothoracic surgery department for surgical intervention and further evaluation. The mass was removed completely (fig-3). Histopathologic examination of the tumor confirmed the diagnosis of atrial Myxoma (Macroscopic measurement showed $5.5 \times 5 \times 3.5 \mathrm{~cm}$ in size) (fig-3). The tumor extends along stained surgical margins and non-diffusely infiltrates striated muscle cells of the myocardium with low-grade myxoid change. On a postoperative day 10 , the patient was discharged to his home on foot. One year later, a control echocardiography and enhanced CT scan was performed and no abnormalities were detected.

\section{Discussion:}

Myxomas are a benign cardiac tumor that is usually involved in the left atrium that occurs most often in female patients aged between 30 to 70 , and more than half of all primary cardiac neoplasms are Myxomas [3]. Primary cardiac tumors in the pediatric age group are rare. 
The most clinical manifestations of patients with cardiac myxoma are dyspnea, followed by chest pain, syncope, sudden death, embolic events, cardiac arrhythmias, and superior vena cava obstruction [4].In our patient presented with syncope and dyspnea on exertion that stated that develops while he is sweeping and lost his consciousness.

Echocardiography is the most reliable method for the diagnosis of myxomas, and it's reliable and easy for differential diagnosis and decision-making for the treatment of such patients [5]. In the present case, Echocardiography showed a tremendous, polypoid, nonhomogeneous left atrial mass with an irregular surface.

In conclusion, Cardiac Myxoma can cause left atrial obstruction and systemic embolization. So early diagnosis with surgical intervention is the definitive management of the tumor and should not be delayed.

\section{Declarations}

\section{Acknowledgements:}

We thank the patient who gave her consent to be publioshed in this case report.

\section{Authors' contributions:}

Mohamed MYM brought the idea of the case study, performed data collection, and prepared the manuscript. Said AA participated in the manuscript preparation and flow up of the patients condition. Mohamed AM participated in the manuscript preparation and perform echocardiography for the diagnose. All authors read and approved the manuscript before submitting.

\section{Funding:}

WE declare that we have no funding source.

\section{Availability of data and materials:}

The data is available from the corresponding author and can be accessed if requested.

\section{Declarations}

\section{Ethics approval and consent to participate:}

This case study was reviewed and approved by Mogadishu Somali Turkish Training and Research Hospital ethic committee. Written consent had obtained from the patient.

\section{Consent for publication:}

All authors gave their consent for this case study to be published. 


\section{Competing interests:}

We declare that we have any no competing of interest.

\section{Author details:}

Mohamed Farah Yusuf Mohamud, Mogadishu Somali Turkish Training and Research Hospital, Mogadishu, Somalia. Email: m.qadar59@gmail.com. Tell No: +252615591689.

Said Abdirahman Ahmed, Mogadishu Somali Turkish Training and Research Hospital, Mogadishu, Somalia. Email:saciidcabdi114@gmail.com Tell No: +252615583381.

Mohamed Abdullahi Mohamud, Mogadishu Somali Turkish Training and Research Hospital, Mogadishu, Somalia. Email: maxamadf10@gmail.com. Tell No: +252616983961.

\section{References}

1. Virmani R, Burke A, Farb A. Atlas of cardiovascular pathology. WB Saunders Company; 1996.

2. Al-Mamgani A, Baartman L, Baaijens M, de Pree I, Incrocci L, Levendag PC. Cardiac metastases. International journal of clinical oncology. 2008 Aug 1;13(4):369.

3. Demir M, Akpinar O, Acarturk E. Atrial myxoma: an unusual cause of myocardial infarction. Tex Heart Inst J. 2005;32(3):445.

4. Simpson L, Kumar SK, Okuno SH, Schaff HV, Porrata LF, Buckner JC, Moynihan TJ. Malignant primary cardiac tumors: review of a single institution experience. Cancer: Interdisciplinary International Journal of the American Cancer Society. 2008 Jun 1;112(11):2440-6.

5. Ha JW, Kang WC, Chung N, Chang BC, Rim SJ, Kwon JW, Jang Y, Shim WH, Cho SY, Kim SS, Cho SH. Echocardiographic and morphologic characteristics of left atrial myxoma and their relation to systemic embolism. American Journal of Cardiology. 1999 Jun 1;83(11):1579-82.

\section{Figures}




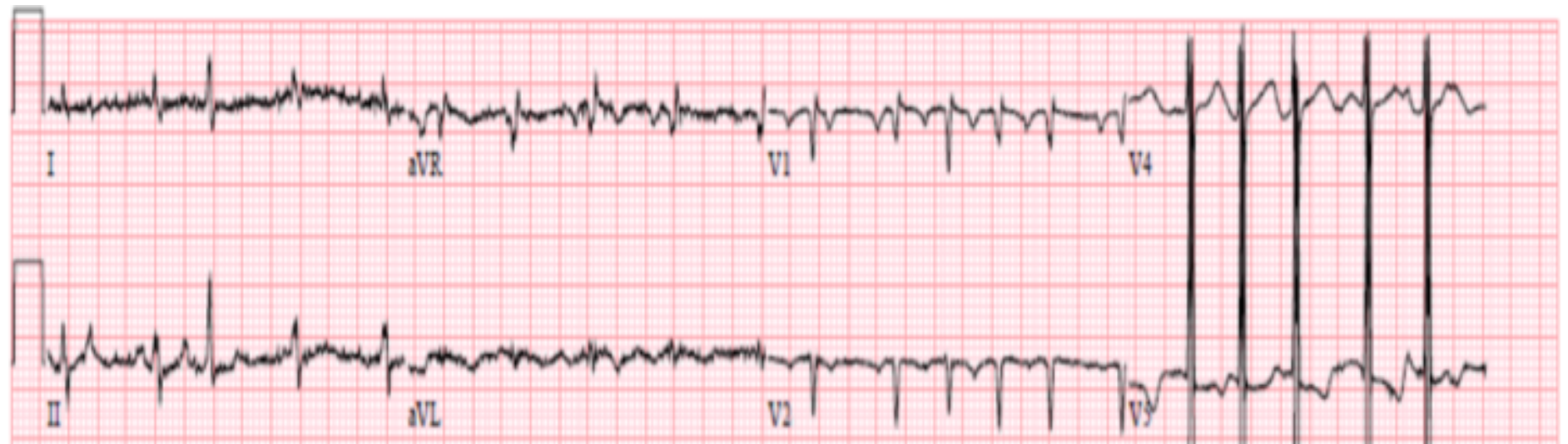

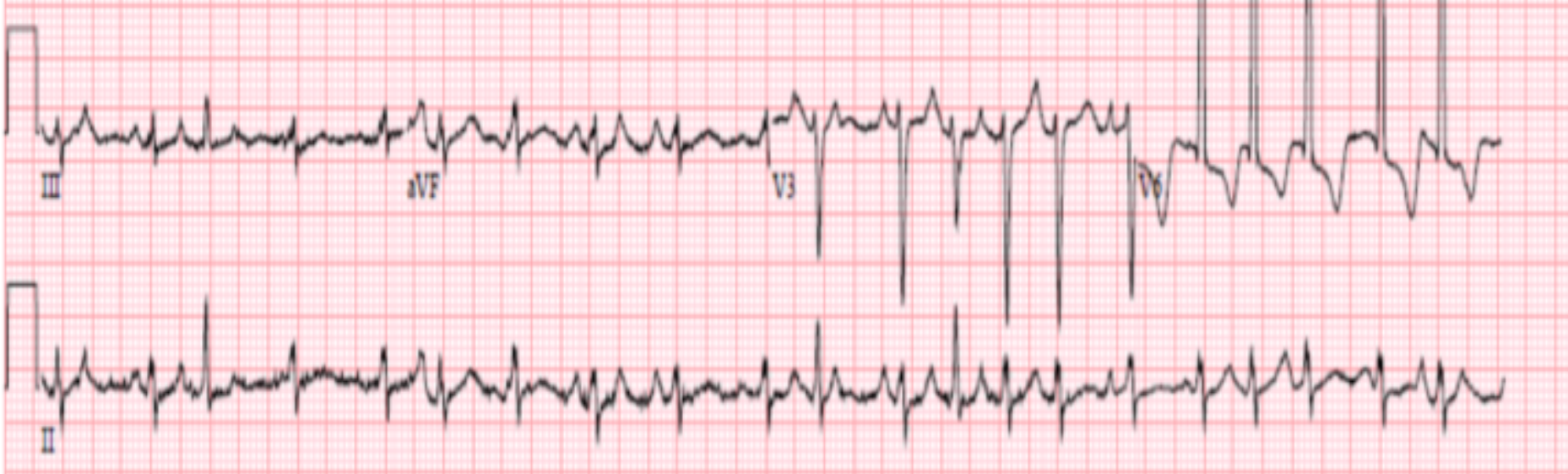

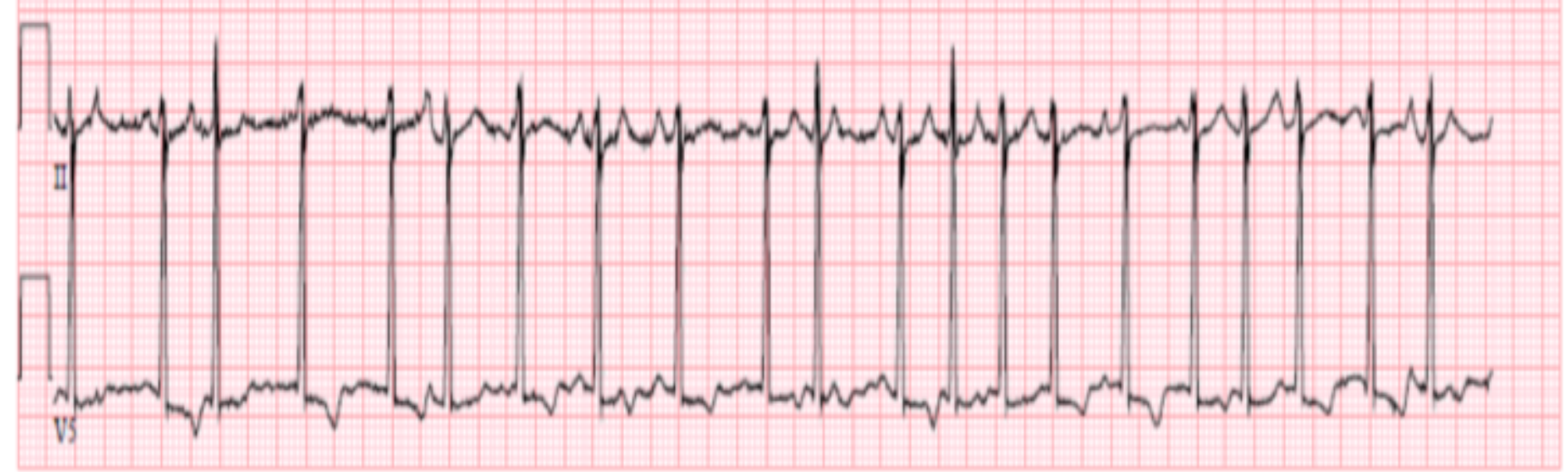

Figure 1

Electrocardiogram shows left atrial enlargement. 

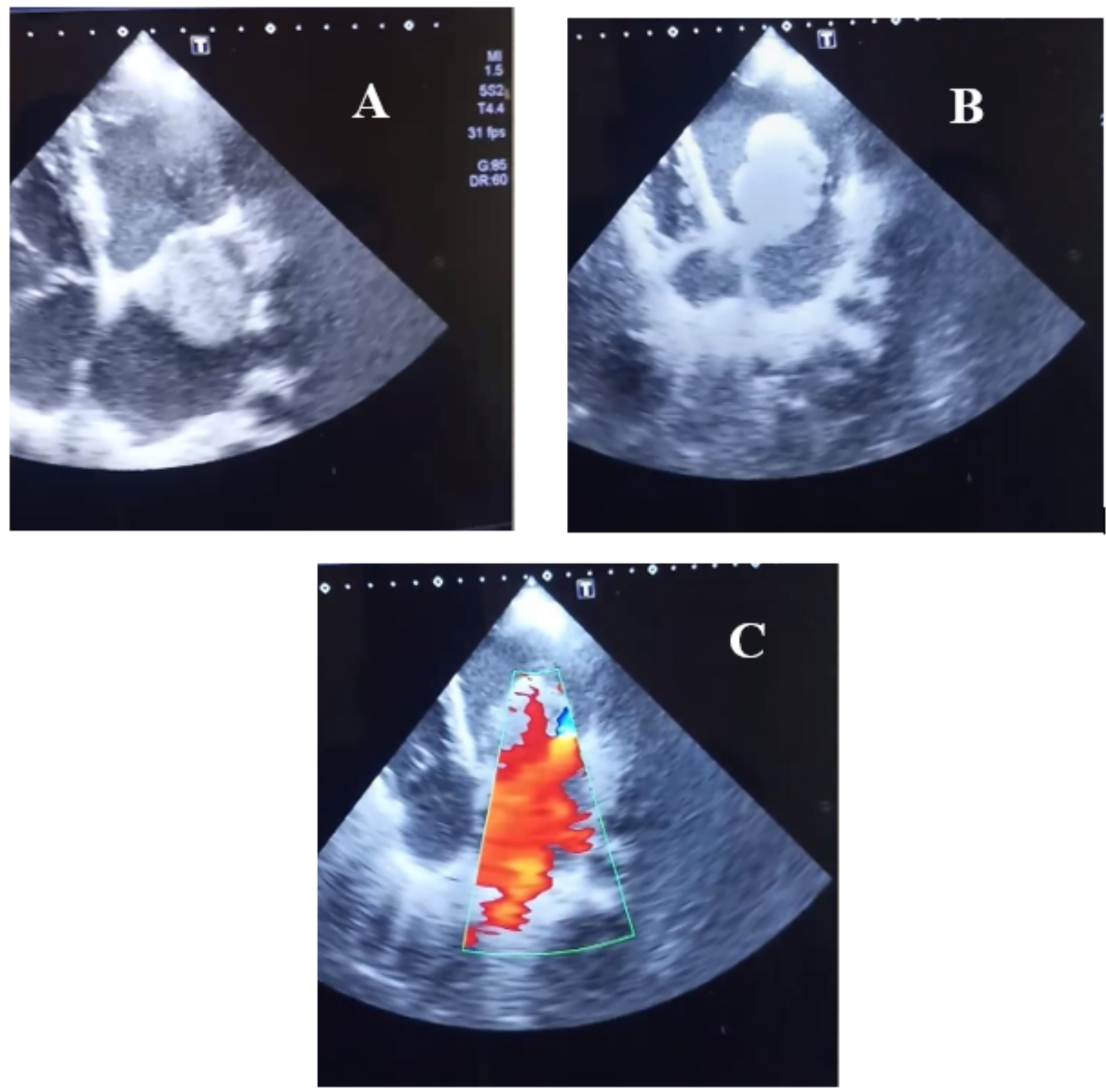

Figure 2

Echocardiography reveals a large, non-homogeneous irregular mass at the level of the mitral valve. 


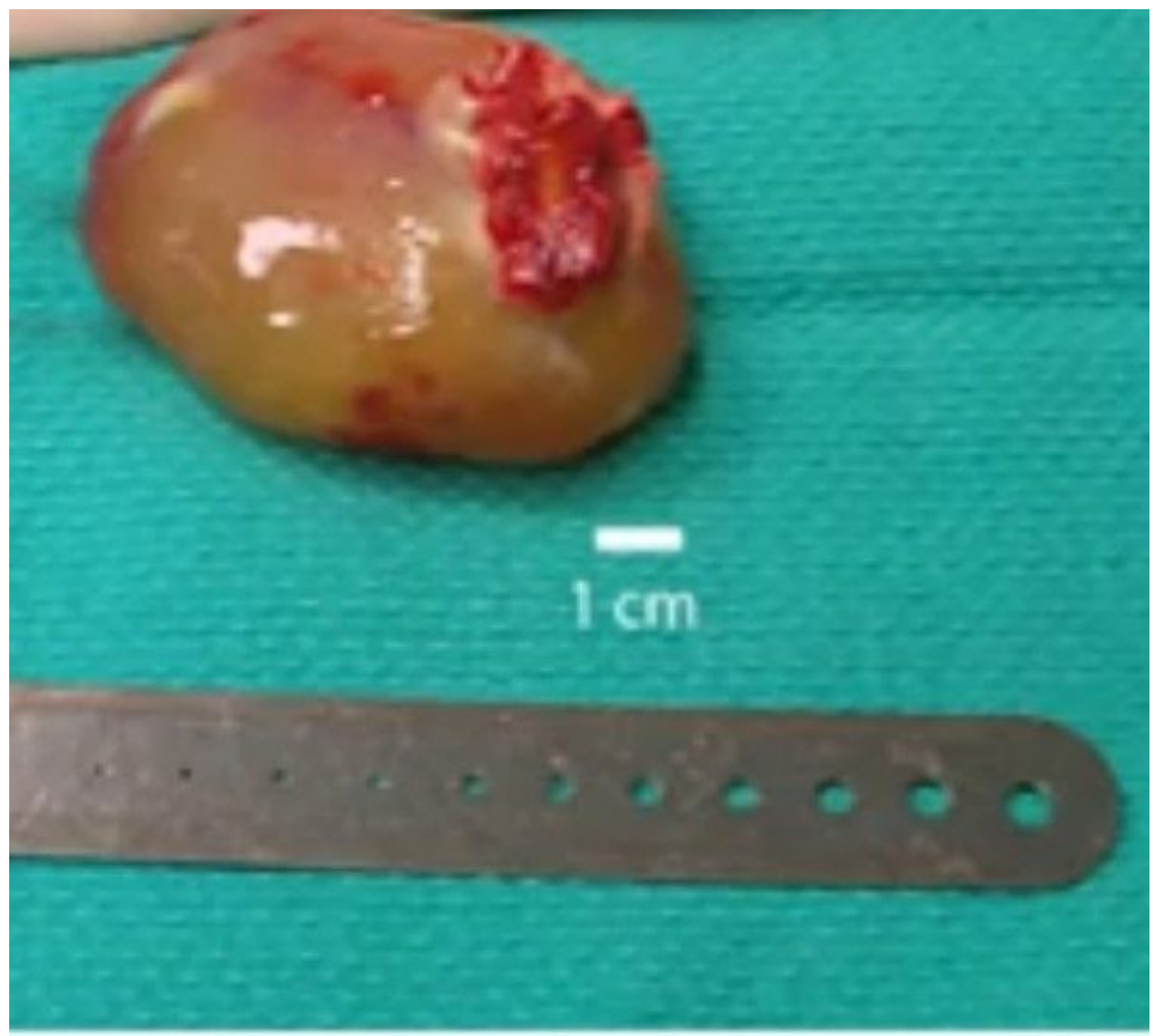

\section{Figure 3}

Macroscopic appearance of the cross-section resected mass with a measurement of $5.5 \times 5 \times 3.5 \mathrm{~cm}$ in size, with relatively smooth contours, with non-diffusely hemorrhagic slice surface, completely consisting of an off-yellow tumor. 